\title{
Generation of PWM Based on Simple Relay Feedback Systems
}

\author{
Abdelouahab ZAATRI ${ }^{1}$, Ridha KELAIAIA ${ }^{2}$ \\ ${ }^{1}$ Laboratory of Applications of Advanced Technology (LATA), University of Constantine1, \\ Constantine, Algeria, e-mail: azaatri@yahoo.com \\ ${ }^{2}$ LGMM Laboratory, College of Technology, Université 20 Août 1955-Skikda, Algeria, \\ e-mail: r.kelaiaia@univ-skikda.dz
}

Manuscript received June 02, 2020; revised November 18, 2020.

\begin{abstract}
This paper is concerned with the analysis of the tracking capability of Relay Feedback Control Systems (RFCS). The envisioned application is the generation of PulseWidth-Modulation (PWM) with variable duty-cycle and frequency known also as DutyCycle Modulation. In general, two main issues have to be solved with RFCS when used for regulation and tracking. The first issue concerns the determination of autonomous self-oscillations which are generated by the closed loop. The second issue concerns the determination of the relationship between the reference and the output signals. However, if the first issue has beneficiated from different approaches and has been solved particularly in case of zero reference signal, the second issue has got insufficient theoretical results because of mathematical difficulties for obtaining analytical expressions. Considering these reasons, a deep analysis of RFCS constituted of a hysteretic relay and linear systems of first order is presented. Analytical expressions relating self-oscillations and output mean values with respect to the reference signals are obtained- The analysis of these relationships led to infer some conditions under which the relay feedback control can generate commands for regulating and tracking reference signals. It highlights also the key role played by the hysteresis band width for applications based on RFCS. Our analysis, exploiting the analytical expressions, can be used to achieve a complete design for power converters. Moreover, simulation and experiments have confirmed our results.
\end{abstract}

Keywords: Relay feedback, On/Off control, PWM, tracking.

\section{General information}

RFCS have found place in many applications such as servomechanisms, trackers, power converters, sliding mode control systems, relay auto-tuners, sigma-delta modulators, etc. [1], [2]. In fact, by design, as other type of negative 
feedback control systems, RFCS can be essentially considered as regulators or trackers since their outputs should follow their reference inputs as close as possible. In case of a constant reference signal set-point or desired signal, the problem corresponds to regulators. In case where the reference is a time variable signal or trajectory, the problem corresponds to a tracking one which is known as a servo problem.

It is well known that the most important characteristic of RFCS is their capability to autonomously generate stable self-oscillations (limit cycles) even without input excitations. Many systems are close to RFCS as they use switching mode called also "Bang-Bang" and "on-off" mode such as pulse-width modulators, sliding mode controllers, time optimal control systems, etc. Some theories based on state-space temporal approaches as well as harmonic approaches were developed to analyze RFCS. In effect, there are many approaches that enable to determine or approximate the self-oscillations that rise in these systems and their stability [1-4]. However, as noticed in [5], the vast majority of publications devoted to RFCS have focused on the analysis of the parameters of the oscillations and their stability. Conversely, the input-output problems did not produce enough results certainly because of mathematical difficulties in expliciting input-output relationships. For this reason, the simulation and the realization of real systems constitute alternatives to analyze input-output problems.

In a previous paper [6], [7], we have designed and realized some experimental prototypes of RFCS for designing and implementing power converters. We have studied and performed both simulations and experiments which have shown a tracking capability of the output with respect to the desired input. These systems revealed to be able to generate autonomously a pulse width modulation (PWM) with variable frequency.

In this paper, we present a deep analysis of these systems especially with a first order linear system. Based on the obtained analytical results, we infer the conditions under which these systems can be used as regulators and trackers.

\section{Model of RFCS}

\section{A. The proposed model}

There are many basic configurations of RFCS. We consider one type of model which is schematically presented in Fig.1. It has been already described in [6] and [7]. This system is constituted by a closed loop including in the forward path a symmetrical relay with a hysteresis band width noted $2 \cdot h$ and an output signal amplitude noted $E$. In the feedback path, it includes a linear first order system 
with transfer function $H(s)$. The closed loop is followed by a linear system of second order or higher noted $G(s)$.

$G(s)$ is designed to filter the high frequencies contained in the on-off signals, and to extract the mean value which represents the tracking value. The involved variables are: the reference signal which is the input of the closed loop $r(t)$ to be tracked, the control signal $x(t)$ which represents the error $(x(t)=r(t)-z(t))$, the controlled signal $u(t)$, the feedback signal $z(t)$, and the output signal $v(t)$.

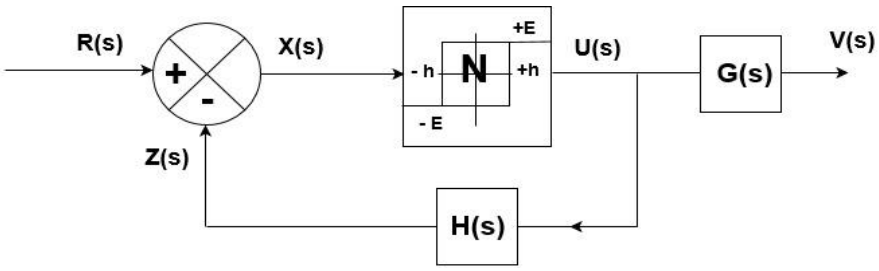

Figure 1: Model of an RFCS.

\section{B. The behavior of the relay " $N$ "}

For the sake of simplicity, we assume that $x(t)$ the input signal of the relay has a triangular shape and can cross the threshold values $( \pm h)$ at some specific instants $t_{k}$. Then, the relay output $u(t)$ will be a succession of alternating pulses of sign $( \pm E)$. The instants during which the jumps occur are called the switching instants $t_{k}$. These instants are determined by the following conditions:

$$
\left\{\begin{array}{lll}
\text { if } \quad\left(x(t)>+h \text { and } \frac{d x(t)}{d t}>0\right) & \Rightarrow u(t)=+E \\
\text { if } \quad\left(x(t)<-h \text { and } \frac{d x(t)}{d t}<0\right) & \Rightarrow u(t)=-E
\end{array}\right.
$$

with $x(t)=r(t)-z(t)$.

\section{Tracking signals with RFCS}

To illustrate the tracking capability of the RCFS, we will develop and analyze the relationship between the mean value of the output of the linear system (the tracker) and the reference input (the tracked signal). The elaboration of commands is automatically generated through the closed loop according to the conditions specified in (1). The commands are expected to be a sequel of On-Off state intervals generated by the relay output. It is well known that this 
configuration of RFCS acts as a pulse with modulator (PWM), where the tracked information is contained in the On and Off durations of the relay impulses. Therefore, determination of these durations is a fundamental problem for designing applications based on this technique.

\section{A. Determination of switching times}

Consider the electrical circuit containing a resistor $R$ and a capacitor $C$ as presented in Fig. 2. It is a linear system of first order which corresponds to the system with transfer function $H(s)$ in Fig. 1. The input and the output of this system are respectively noted $u(t)$ and $z(t)$ according to Fig. 2 .

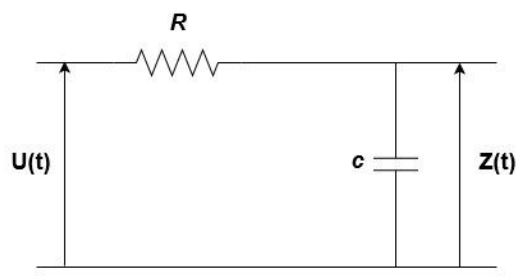

Figure 2: Linear filter of first order.

If the input signal of the relay feedback control is null, then, the closed loop generates a stable symmetrical self-oscillation of a square shape with a null mean value of the output $u(t)$ and $z(t)$. Thus, the only relevant problem is the determination of the frequency of the generated stable self-oscillations. For this purpose, many approaches have been proposed [3], [4], [5]. However, if the input signal to be tracked $r(t)$ is not null, the used approaches assume its slow variation compared to the on-off durations generated by the output relay. In this case, the analysis will be simplified by assuming that the input signal is constant: $r(t) \approx r_{0}$.

For the first order filter of Fig. 2, the output is a solution of the following differential equation:

$$
\tau \cdot \frac{\mathrm{d} z_{k}}{\mathrm{~d} t}+z_{k}=u(t)=(-1)^{k \cdot} \cdot E .
$$

The subscript $k$ denotes the intervals of commutation or pulse durations.

In case of a constant reference signal $r(t)=r_{0}$, the signal $u(t)$ is a sequence of unsymmetrical pulses taking alternately the values $u(t)= \pm E$ in a rectangular shape. To determine the switching instants and the durations of the control impulses, we proceed by integrating equation (2) under conditions (1). 
B. Determination of pulse durations for a constant reference signal

By admitting the piecewise continuity over any time interval limited by two successive switching instants $t_{k}$ and $t_{k+1}(k=0,1,2, \ldots \ldots n)$, the integration of (2) under conditions (1) permits to determine the analytical results concerning the On and Off durations and consequently the period of the self-oscillation which are presented in [6].

To determine the pulse durations for a constant reference signal, let's consider Fig. 3, which represents the temporal evolution of the input $u(t)$ and output $z(t)$ of the linear filter described by equation (2).

If we consider intervals $k$ which are even, the solution of (2) can be written in the form: $z(t)=z_{0} \cdot e^{-t / \tau}+E$.

The instants of commutation during these intervals happen according to (1):

$$
\begin{gathered}
z(0)=z_{0}+E=r_{0}-h, \\
z\left(T_{1}\right)=z_{0} \cdot e^{-T_{1} / \tau}+E=r_{0}+h,
\end{gathered}
$$

where $T_{1}$ is the duration of positive impulses.

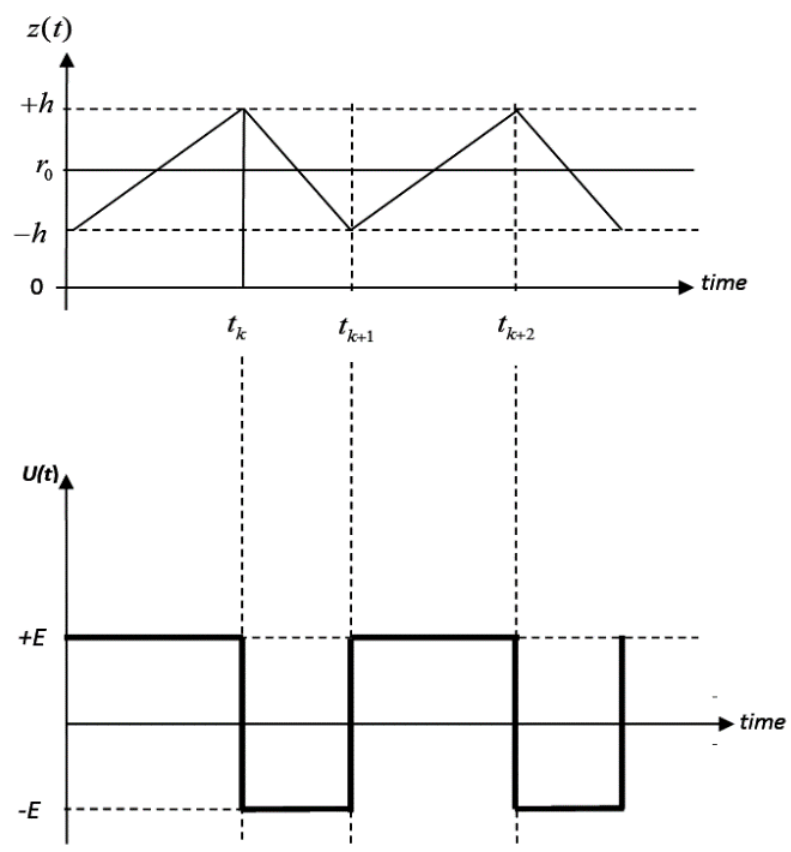

Figure 3: The input and output signals $z(t), u(t)$ 
By combining (3a) and (3b), we get:

$$
\frac{T_{1}}{\tau}=\ln \left(\frac{E-r_{0}+h}{E-r_{0}-h}\right)=\ln \left(\frac{1+\frac{h}{E-r_{0}}}{1-\frac{h}{E-r_{0}}}\right)=2 \cdot \operatorname{argth}\left(\frac{h}{E-r_{0}}\right)
$$

If we consider intervals $k$ which are odd, the solution of (2) can be written in the form: $z(t)=z_{0} \cdot e^{-t / \tau}-E$. The instants of commutation during this interval happen according to (1).

$$
\begin{gathered}
z(0)=z_{0}-E=r_{0}+h, \\
z\left(T_{2}\right)=z_{0} \cdot e^{-T_{2} / \tau}-E=r_{0}-h .
\end{gathered}
$$

$T_{2}$ is the duration of negative impulses. By combining (5a) and (5b), we get:

$$
\frac{T_{2}}{\tau}=\ln \left(\frac{E+r_{0}+h}{E+r_{0}-h}\right)=\ln \left(\frac{1+\frac{h}{E+r_{0}}}{1-\frac{h}{E+r_{0}}}\right)=2 \cdot \arg \operatorname{th}\left(\frac{h}{E+r_{0}}\right) .
$$

The pulse duration $(\mathrm{ON}), T_{1}$ can be expressed as:

$$
T_{1}=t_{k+1}-t_{k}=2 \cdot \tau \cdot \arg \operatorname{th}\left(\frac{h}{E-r_{0}}\right) \text { under the condition } h<\left|E-r_{0}\right| .
$$

The pulse duration (OFF), noted $T_{2}$ can be expressed as (4):

$$
T_{2}=t_{k+2}-t_{k+1}=2 \cdot \tau \cdot \arg \operatorname{th}\left(\frac{h}{E+r_{0}}\right) \text { under the condition } h<\left|E+r_{0}\right| \cdot
$$

By manipulating the relations (7) and (8), it follows that for a constant reference input value $r_{0}$, the generated command $u(t)$ is a periodic signal of rectangular shape, with period $T=T_{1}+T_{2}$, which can be expressed as follows:

$$
T=2 \cdot \tau \cdot \arg \operatorname{th}\left(\frac{2 \cdot h \cdot E}{E^{2}-r_{0}^{2}+h^{2}}\right) \text {. }
$$




\section{Comments:}

- The obtained expressions (7), (8) and (9) are functions of the constitutive parameters of the system $(h, E, \tau)$, and of the reference signal $r_{0}$. We can observe that $T_{1}, T_{2}$ and $T$ are biased by the value of the input signal $r_{0}$, which causes the dissymmetry of the commands. This fact reveals that the obtained PWM is of variable frequency.

We can notice that similar results have been obtained by applying the theory of Locus of a Perturbed Relay System (LPRS) [5]. Under the approximation of slow variation of the reference signal with respect to the period of self-oscillation, comparable results have been also obtained by [8].

- If we consider $r_{0}=0$ and $h<<E$ in (9), then we can notice that the period $T$ reduces to $T_{0}$.

$$
T_{0}=4 \cdot \tau \cdot \arg \operatorname{th}\left(\frac{h}{E}\right) .
$$

This means that if $r(t)=r_{0}=0$, the RFCS generates a self-oscillation which corresponds to a square-shaped signal of period $T_{0}$. Furthermore, this period $T_{0}$ is the smallest period the system generates and therefore corresponds to the maximum frequency the relay feedback can generate under the given configuration.

- Fig. $4 a$ and Fig. $4 b$ present the variation of the period and of the frequency of the self-oscillation w.r.t the reference input according to relation (7) and its reciprocal. These figures have been obtained for the parameters: $E=12$ volts, $h=1, \tau=10 \mathrm{~s}$. We can observe from these figures that the period of selfoscillation increases in a quasi-quadratic manner with the increase of the reference input $r_{0}$ (see Fig. $4 a$ ). Inversely, the frequency of this self-oscillation decreases (Fig. 4b). However, we notice the presence of a singularity which is reflected by a discontinuity in Fig. $4 a$ and Fig. $4 b$. This singularity occurs when the argument of the inverse of tangent hyperbolic function argth in (9) tends to unity, leading the period $T$ to infinity. This corresponds to $\left(\frac{2 \cdot h \cdot E}{E^{2}-r \cdot o^{2}+h^{2}}\right)=1$.

This leads to a condition that limits the maximal value of the reference signal which corresponds to the inequality:

$$
\left|r_{0}^{\max }\right|<E-h .
$$


Near this point of singularity $\left(r_{0}^{\max }=E-h\right)$, the RFCS can generate only very low frequencies. Therefore, the system cannot follow the reference signal and is useless in this region.

- There are also technical limitations for high frequencies imposed by the finite switching time ( $\tau_{\text {on }}$ and $\tau_{\text {off }}$ ) of the used switching elements such as electromechanic or electronic devices [7]. This fact leads to additional constraints like the following one which imposes that the pulse duration should be larger than the switching time of the used relays and switches. It can be expressed by the following expression:

$$
T_{1}=2 \cdot \tau \cdot \arg \operatorname{th}\left(\frac{h}{E-\left|r_{0}\right|}\right) \gg>\max \left(\tau_{o n}, \tau_{o f}\right) .
$$

Thus, the general condition to obtain a PWM is to impose that the minimal intervals of commutation should be much larger than the switching times of the relays and the used switches: $\min \left(T_{1}, T_{2}\right) \square \max \left(t_{\text {on }}, t_{\text {off }}\right)$.

By observing Fig. $4 a$ and Fig. $4 b$ for different values of $h$, we conclude that the smaller the width of the hysteresis the more we avoid the singularity and the more the generated PWM satisfies the application requirements.

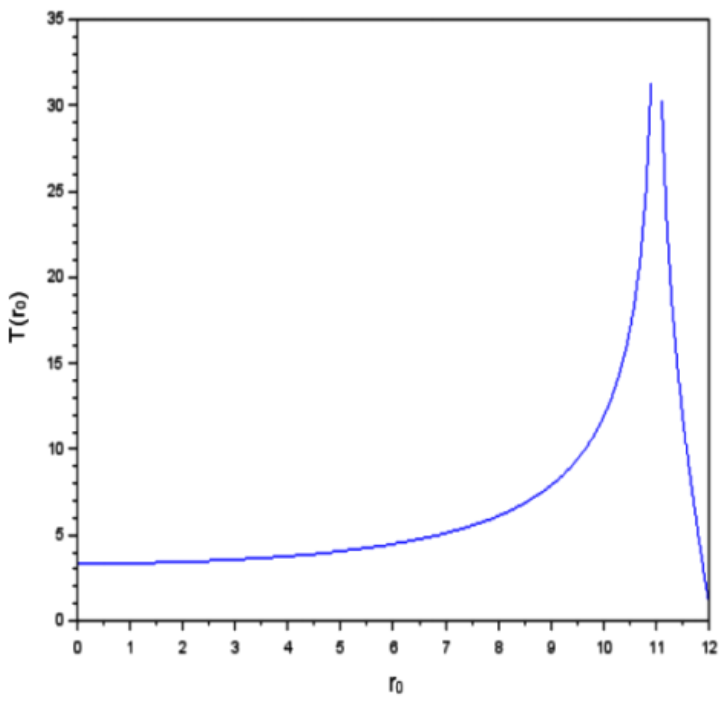

4a: Period 


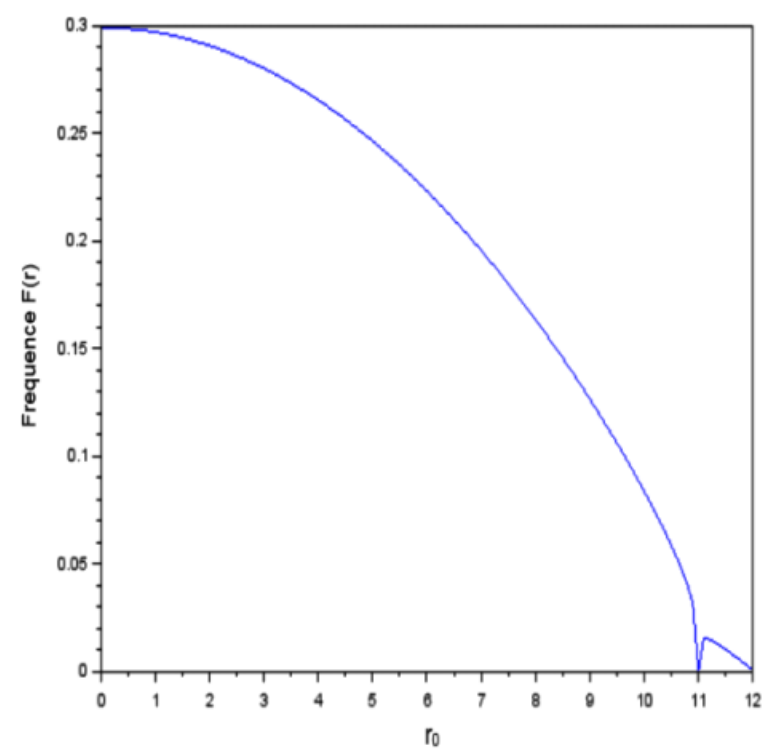

$4 b$ : Frequency of self-oscillation

Figure. 4: Curves of the period and the frequency of self-oscillation w.r.t. reference signal

\section{Determination of the output mean value $u_{m}\left(r_{0}\right)$}

To consider the tracking capability of our control system, we determine the mean value $u_{m}$ of output $u(t)$, which is the measure of the dissymmetry of the periodic signal. It is determined by the following expression:

$$
u_{m}=E \cdot \frac{\left(T_{1}-T_{2}\right)}{\left(T_{1}+T_{2}\right)} .
$$

By replacing $T_{1}$ and $T_{2}$ from (3) and (4) in (9), the mean value is obtained in the form:

$$
u_{m}\left(r_{0}\right)=\frac{\operatorname{argth}\left(\frac{2 \cdot h \cdot E}{E^{2}-r_{0}^{2}-h^{2}}\right)}{\operatorname{argth}\left(\frac{2 \cdot h \cdot E}{E^{2}-r_{0}^{2}+h^{2}}\right)} \cdot r_{0} .
$$


- This expression highlights the relationship between the average value $u_{m}$ of $u(t)$ and the target $r_{0}$ as well as the relay parameters of the RFCS $(h, E)$. It is noteworthy to notice that it is independent from the time constant $(\tau)$ of the linear system. We notice also that this relationship is almost linear or quasi-linear if condition (11) is fulfilled. This means that the tracking of $r_{0}$ by $u(t)$ is effective as long as this condition holds. (see Fig. 4).

- Nevertheless, the analysis of expression (14) reveals the presence of two points of singularity that lead to discontinuities, one in the argument of the numerator and the second in the denominator. The first discontinuity occurs when the argument in the numerator $\left(\frac{2 \cdot h \cdot E}{E_{0}^{2}-r_{0}^{2}-h^{2}}\right)$ tends to unity. It imposes a discontinuity in the period of self-oscillation that corresponds to a value of the reference variable:

$$
\left|r_{01}\right|=\sqrt{E^{2}-2 \cdot h \cdot E-h^{2}}=\sqrt{(E-h)^{2}-2 \cdot h^{2}} .
$$

Similarly, the term in the denominator leads also to a discontinuity at the value of the reference such as:

$$
\left|r_{02}\right|=E-h .
$$

By comparing $r_{01}$ and $r_{02}$, we have: $r_{01}<r_{02}$. Moreover, the value corresponds to (13), so that: $r_{01}=r_{0}^{\max }$.

Fig. 5 shows the evolution of $u_{m}\left(r_{0}\right)$ obtained for the parameters: $E=12$ volts and $\tau=10 \mathrm{~s}$. To highlight the discontinuities, two curves are superimposed, each parameterized by a value of $h$. The left side curve corresponds to $h=1$ volt while the right side one corresponds to $h=3$ volts .

According to Fig. 5, the RFCS can only track reference signals in the range between $r_{0}=0$ and the vicinity from the left side of the first discontinuity $\left|r_{01}\right|$.

These last results limit seriously the regulating or tracking capability of RFCS. Fig. 5 shows the two discontinuities. Near the vicinity of the discontinuity and beyond, the theoretical model is not applicable.

Again, by observing for different values of $h$, we conclude that the smaller the width of the hysteresis the more the relationship becomes linear and generates PWM that satisfies the application. 


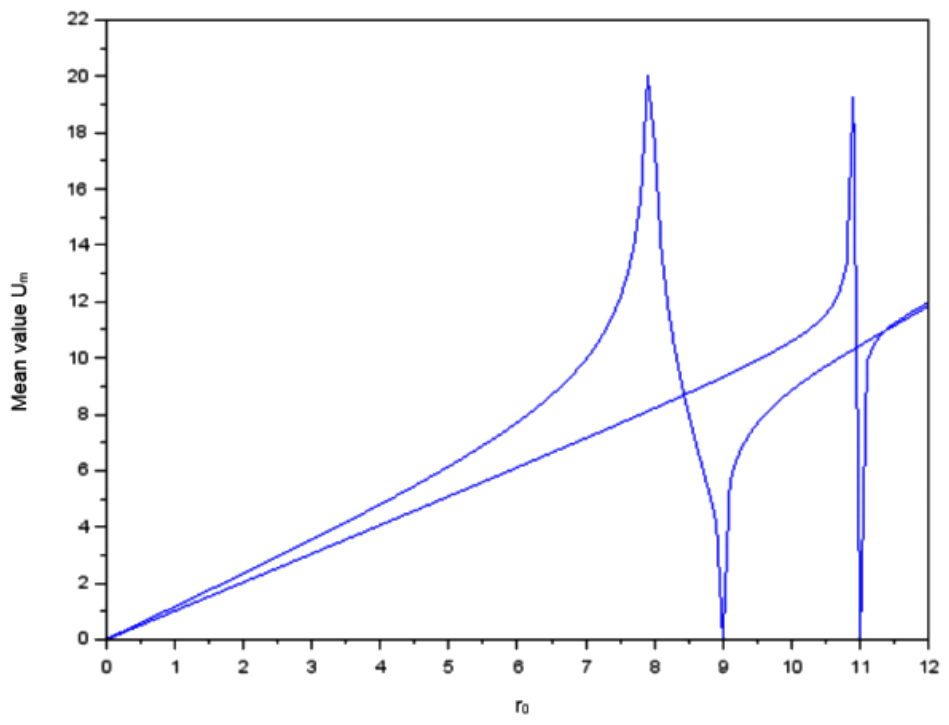

Figure 5: The mean value w.r.t the reference signal $u_{m}\left(r_{0}\right)$.

- On the other hand, if we consider the approximation $\arg \operatorname{th}(x) \cong x$, we can write $u_{m}$ as:

$$
u_{m}\left(r_{0}\right)=\left(\frac{E_{0}^{2}-r_{0}^{2}+h^{2}}{E_{0}^{2}-r_{0}^{2}-h^{2}}\right) \cdot r_{0} .
$$

If we consider $\left(\frac{h}{E-\left|r_{0}\right|}\right)<<1$, the regulation effect of the RFCS appears in the derived expression: $u_{m}\left(r_{0}\right) \cong r_{0}$, which means that the mean value approximately follows the reference signal.

\section{Determination of the Duty Cycle}

We can estimate and highlight the dependency between the duty cycle and the reference signal $r_{0}$. The duty cycle can be defined as: $D\left(f_{0}\right)=\frac{T_{1}}{T}$. By replacing $T_{1}$ and $T$ by their expressions in (7) and (9), we get the duty cycle: 


$$
D\left(r_{0}\right)=\frac{\arg \operatorname{th}\left(\frac{h}{E-r_{0}}\right)}{\arg \operatorname{th}\left(\frac{2 \cdot h \cdot E}{E^{2}-r_{0}^{2}+h^{2}}\right)} .
$$

As the mean value of the relay output, the duty cycle depends on the reference signal and on the relay parameters $(h, E)$. The obtained PWM is thus performed at a variable frequency. If $r_{0}=0$, the duty cycle equals $1 / 2$ which means that $T_{1}$ is half of the period $T$. This corresponds to a relay output of a square shape. The duty cycle w.r.t the reference signal is also subject to two singularities, one in the numerator and the second in the denominator. However, the two singularities are similar and occur if $\left|r_{02}\right|=E-h$, which corresponds to the relation already obtained in (16).

Fig. 6 is obtained for the parameters: $E=12$ volts, $h=1$ volt and $\tau=10 \mathrm{~s}$. It presents the evolution of the duty cycle with respect to the reference signal. We can notice the presence of a singularity that has to be avoided in order to have a useful generation of PWM. By observing Fig. 6, we conclude that the smaller the width of the hysteresis band the more the relationship becomes linear and the generated PWM is capable to regulate the output. This duty cycle is almost linear in a range from $r_{0}$ to $r_{01}$, but near the singularities, the behavior diverges in an exponential way. If $\frac{h}{E-\left|r_{0}\right|}<<1$, the duty cycle can be approximated by the following expression:

$$
D\left(r_{0}\right)=\frac{T_{1}}{T} \cong \frac{1}{2 \cdot E} \cdot\left(E+r_{0}\right) .
$$

Expression (19) shows a linear relationship between the duty cycle and the reference signal. 


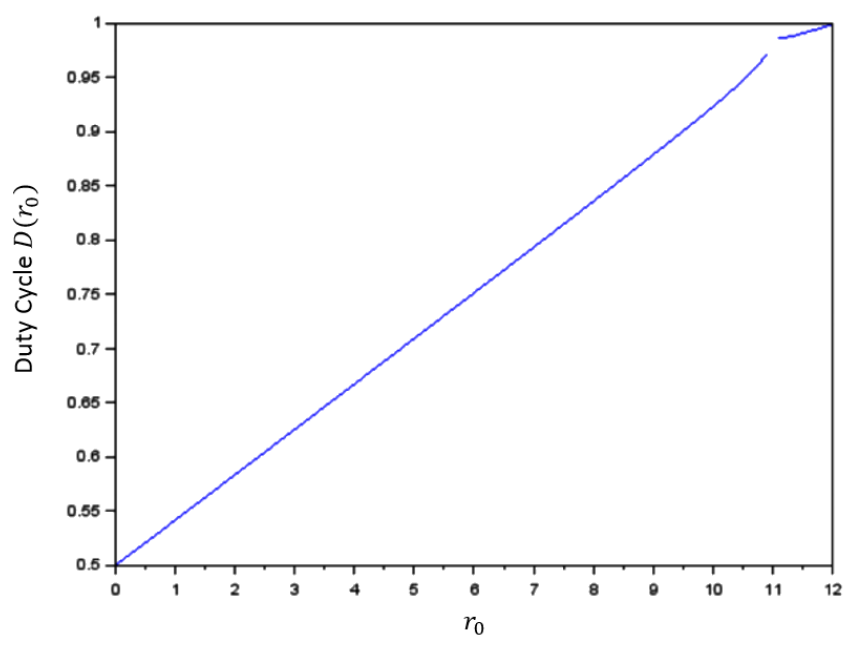

Figure 6: Duty cycle w.r.t reference signal

\section{Special case: reference signal $r(t)$ is variable}

The case when reference signal is time variable corresponds to a tracking case. Analytical results are not easy to obtain. However, the obtained results for pulse durations in case of a constant value of the reference signal can be generalized under the assumption of a slow variation of the reference signal with respect to pulse durations. This way, we can consider $r(t)$ as constant compared to pulse durations and then the obtained results can be locally generalized. Thus, if we approximate the pulse durations $T_{1}(r(t))$ and $T_{2}(r(t))$, then we can infer approximations for the period $T(r(t))$ and the frequency $F(r(t))$ of self oscillations, the mean value $u_{m}(r(t))$ and the duty cycle $D(r(t))$ which can be expressed as follows:

$$
\begin{gathered}
T_{1}(r(t))=2 \cdot \tau \cdot \arg \operatorname{th}\left(\frac{h}{E-r(t)}\right), \\
T_{2}(r(t))=2 \cdot \tau \cdot \arg \operatorname{th}\left(\frac{h}{E+r(t)}\right), \\
T(r(t))=2 \cdot \tau \cdot \arg \operatorname{th}\left(\frac{2 \cdot h \cdot E}{E^{2}+h^{2}-r^{2}(t)}\right),
\end{gathered}
$$




$$
F(r(t))=\frac{1}{T((r(t))}=\frac{1}{2 \cdot \tau} \cdot \frac{1}{\arg \operatorname{th}\left(\frac{2 \cdot h \cdot E}{E^{2}+h^{2}-r^{2}(t)}\right)} .
$$

With a similar configuration of an RFCS used as a DC/AC PWM with variable frequency inverter, under the assumption of slow variation of the reference signal compared to pulse durations, similar results to our approach have been obtained by [8]. As known in PWM systems, the tracking capability of our control system is expressed in the relationship of the mean value of the output signal $u(t)$ relatively to the reference signal. So, let's consider the $u_{m}(r(t))$.

$$
u_{m}(r(t))=\frac{\operatorname{argth}\left(\frac{2 \cdot h \cdot E}{E^{2}-h^{2}-r^{2}(t)}\right)}{\operatorname{argth}\left(\frac{2 \cdot h \cdot E}{E^{2}+h^{2}-r^{2}(t)}\right)} \cdot r(t) .
$$

This can be written in the following form:

$$
u_{m}(r(t))=K(r(t)) \cdot r(t) .
$$

From (24) or (25), we can observe that the tracking of the reference signal is automatically performed independently from its form. The factor $K(r(t))$ represents the distortion that affects the tracking process. With (9) fulfilled, the tracking error decreases if the following condition is satisfied:

$$
\left(\frac{h}{E-\max |r(t)|}\right)<<1 \quad \text { or } \quad h<<E-\max |r(t)| .
$$

This condition enables to approximate (22) by the following expression, which expresses the tracking behavior of the considered RFCS:

$$
u_{m}(r(t)) \cong r(t) .
$$

The same reasoning can be made also in case of the duty cycle.

$$
D(r(t))=\frac{\arg \operatorname{th}\left(\frac{h}{E-r(t)}\right)}{\arg \operatorname{th}\left(\frac{2 \cdot h \cdot E}{E^{2}+h^{2}-r^{2}(t)}\right)} .
$$

If we consider the condition in (26), the duty cycle can be approximated in a 
linear form as

$$
D(r(t)) \equiv \frac{1}{2 \cdot E}(E+r(t)) .
$$

We can see that the tracked information $r(t)$ can be extracted from the duty cycle according to (29) since $r(t)=E \cdot(2 \cdot D(r(t))-1)$.

The relationship between the duty cycle and the reference signal is almost linear.

\section{Results and discussion}

The simulation of the relay feedback control has been performed for various parameters. A model of the studied RFCS with a Simulink implementation using a first order linear system in the feedback and a relay with symmetrical hysteresis is presented in Fig. 6 .

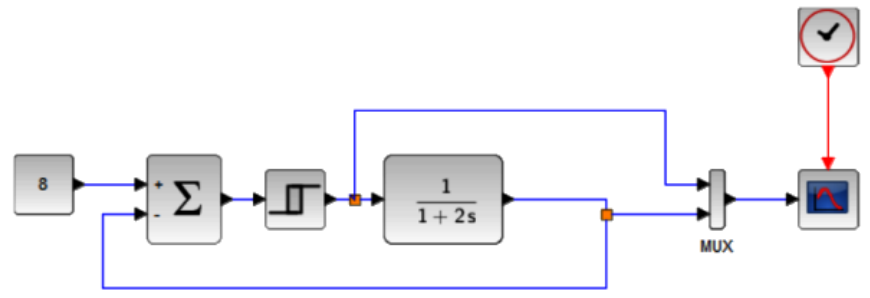

Figure 7: Scheme for simulation

We illustrate this simulation with some results. Fig. 7 shows the adopted model used for generating the PWM related to the application in DC-DC converter control. Some simulation results are presented below. Fig. 8a, Fig. $8 b$ and Fig. $8 \mathrm{c}$ present the output of the relay $u(t)$ in black color and the output of the linear system $z(t)$ with green color. The relay parameters are: $h=1$ volt and $E=10$ volts. The time constant of the linear system is 2 seconds, and the amplification factor equals the unity.

The first group of results concerns the use of the RFCS as a regulator. The results are presented in Fig. $8 a$, Fig. $8 b$ and Fig. $8 c$, for the values of the reference $r_{0}: 0,4$ and 8 Volts, respectively. We can observe that the command is piecewise continuous and switches alternatively between the two extreme values. $u(t)$ belongs to the set $E=10$ volts and $E=-10$ volts. The output is also piecewise continuous and is bounded by the hysteresis limits $( \pm h)$. In the following figures, the output signal $z(t)$ is represented in green color. $z(t)$ tracks the reference 
signal $r_{0}$. We notice the correspondence with the theoretical results. The second group of simulation concerns the use of our RFCS as a tracker of input signals having a sine wave shape.

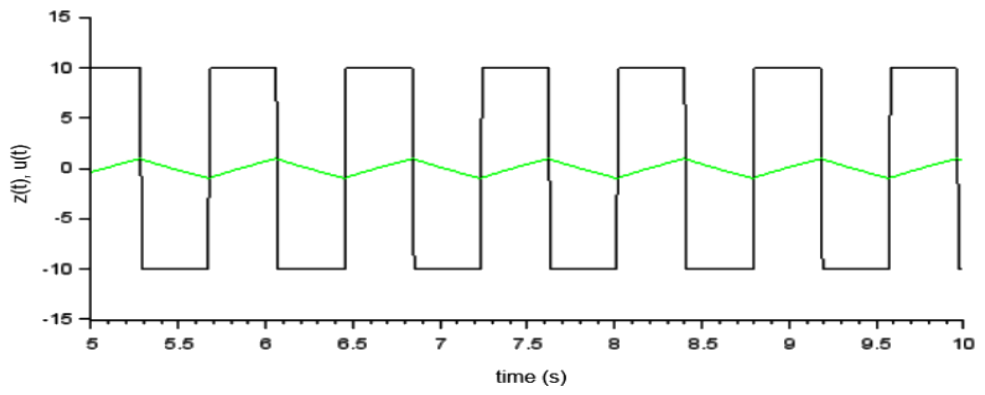

8a: $r_{0}=0$ volts

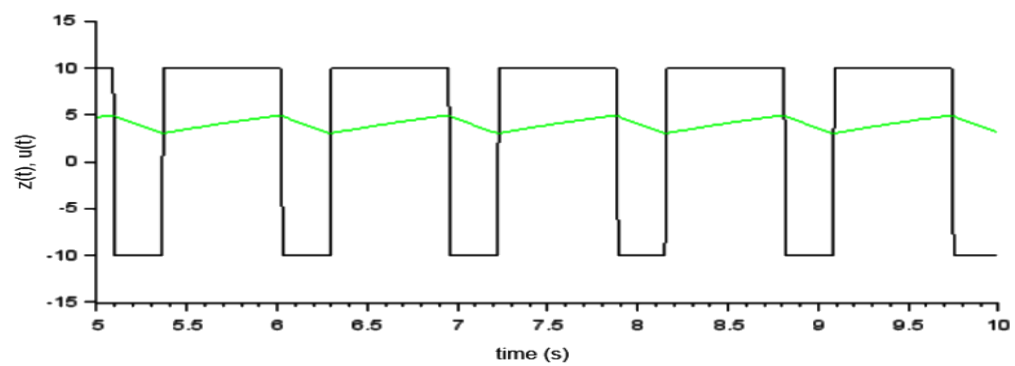

$8 b: r_{0}=4$ volts

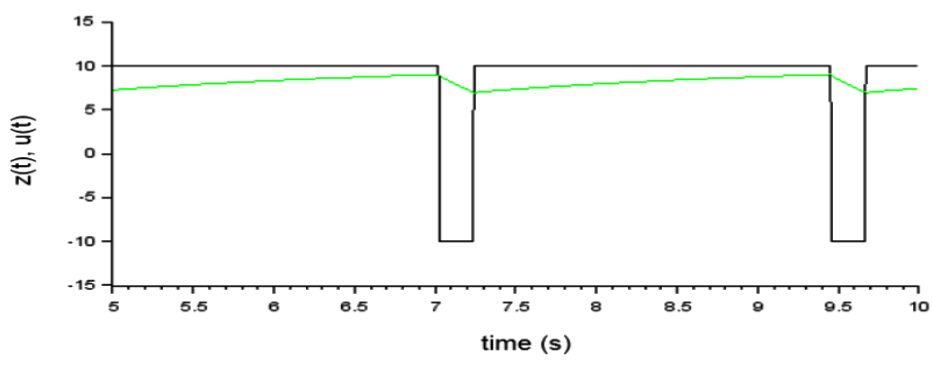

$8 c: r_{0}=8$ Volts

Figure 8: $z(t)$ and $u(t)$ for different values of $r_{0}$

This application corresponds to power inverters DC/AC. The relay parameters are: $h=1$ volt and $E=12$ volts. The time constant of the linear system is 1 second, and the amplification factor equals the unity. Some simulation results are 
presented in Fig. 9a, Fig. $9 b$ and Fig. 9c. They show the relay output $u(t)$ in black color and the linear system output $z(t)$ with green color. The reference signal is a sinewave with an angular frequency $\omega=2 \mathrm{rad} / \mathrm{s}$. The results are obtained for amplitudes which are: 2, 4 and 6 Volts, respectively. The output is also piecewise continuous and is bounded by the hysteresis limits $( \pm h)$. The input $r(t)=r_{0} \cdot \sin (w \cdot t)$ is tracked by the output $z(t)$ which is represented by the green colors in the concerned figures. We notice the correspondence with the theoretical results.

The third group of simulation concerns the use of our RFCS as a tracker of sine signals for different values of the frequency of the reference signal. The relay parameters are: $h=1$ volt and $E=12$ volts. The reference signal is a sine wave with a constant amplitude of 4 Volts and angular frequencies of: 1, 2 and $3 \mathrm{rad} / \mathrm{s}$, respectively. The results are presented in Fig. 10a, Fig. $10 \mathrm{~b}$ and Fig. 10c. We can observe that the command is piecewise continuous and switches alternatively between the two extreme values $u(t)(+E=12$ volts and $E=-12$ volts $)$. The output is also piecewise continuous and is bounded by the hysteresis limits $( \pm h)$. We can observe that the input $r(t)$ is tracked by the output $z(t)$ which is represented by the green color in the concerned figures.

\section{Note:}

An experimental system has been built up. It has been successfully used to test some theoretical results. It has been exploited to generate Pulse Width Modulation (PWM) in a chopper application with feedback through a first order filter. It has also been adapted to the case of a feedback through a second order filter and for tracking various reference signals. For more information and details about these experiments, the interested reader can refer to [6].

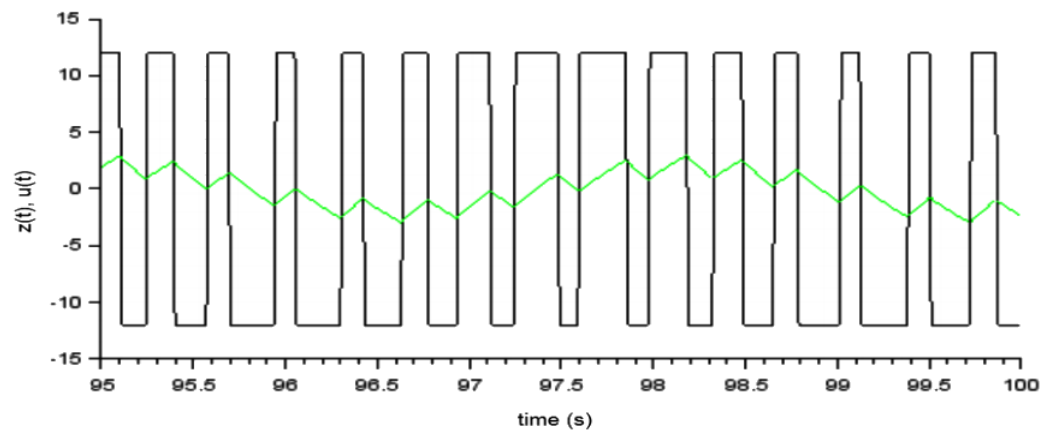

9a: $r_{0}=0$ volts 


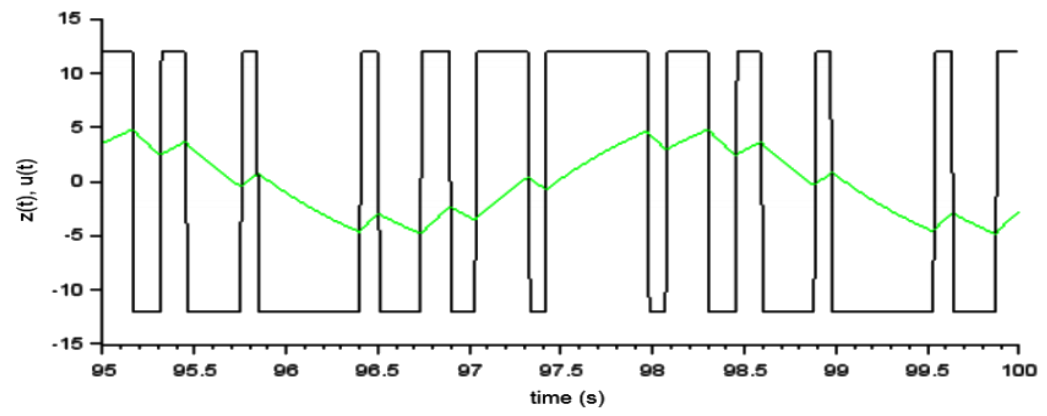

9b: $r_{0}=4$ volts

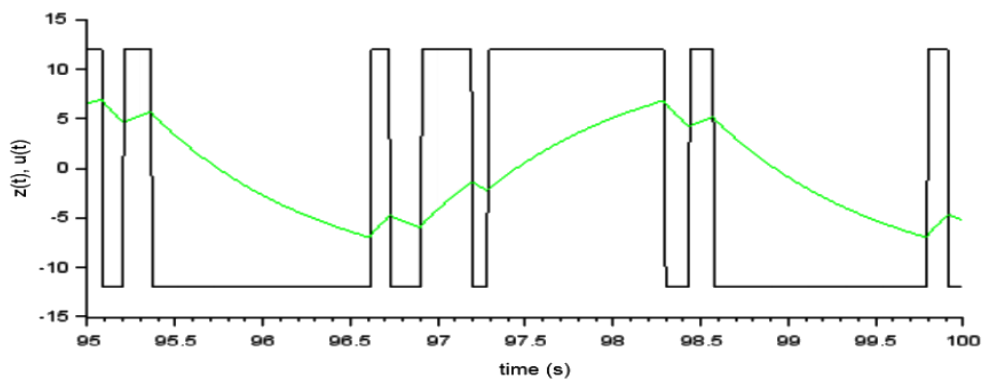

9c: $r_{0}=8$ volts

Figure 9: $z(t)$ and $u(t)$ for $r(t)=r_{0} \cdot \sin (\omega \cdot t)$ with different values of $r_{0}$

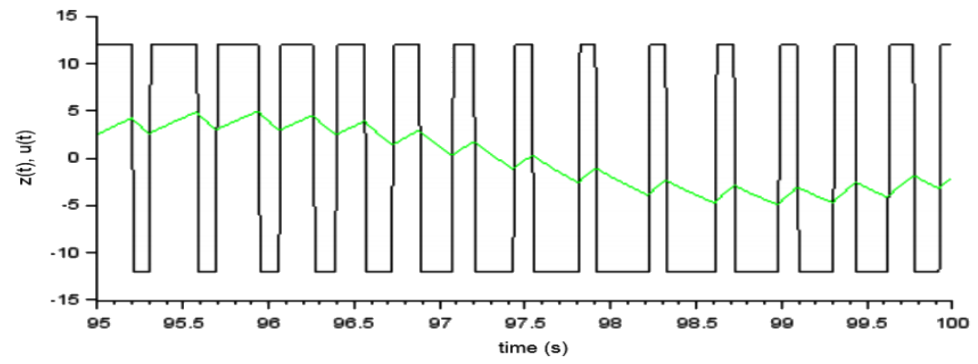

10a: $\omega=1 \mathrm{rad} / \mathrm{s}$ 


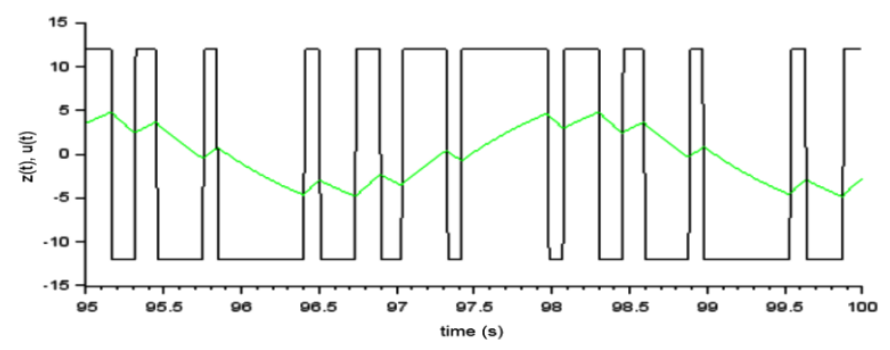

10b: $\omega=2 \mathrm{rad} / \mathrm{s}$

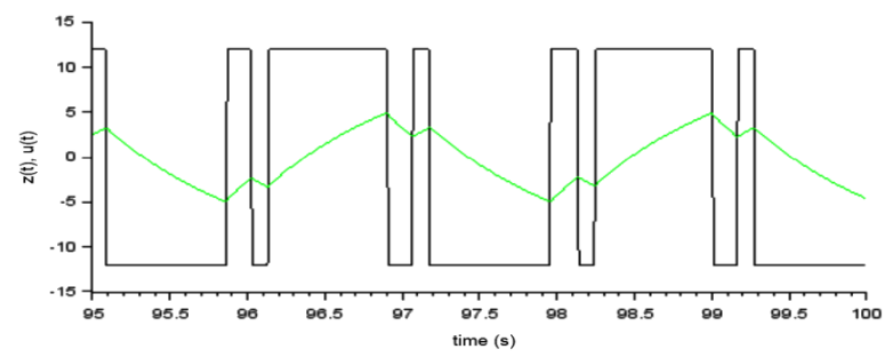

10c: $\omega=3 \mathrm{rad} / \mathrm{s}$

Figure 10: $z(t)$ and $u(t)$ for different values of the frequency

\section{Conclusion}

A study of an RFCS constituted of a relay with symmetrical hysteresis and a linear first order filter feedback circuit has been presented. The pulse durations and the period of self-oscillations in case of constant reference signals have been established. The RFCS generates a PWM with variable frequency and variable duty-cycle. The conditions under which the regulation and the tracking are possible have been analyzed. The conditions for tracking under the assumption that the signal varies slowly in comparison to the pulse durations of the selfoscillations have been extended. Our analysis has led to the conclusion that the width of hysteresis band plays a key role in the design of trackers based on PWM techniques. For applications such as choppers, a complete design can be achieved based on the mathematical expressions obtained in the paper. For DC-AC inverters, the design based on our results is useful but imposes more constraints, since it depends on both the amplitude and the frequency of the sine wave reference signal. For arbitrary signals, the possibility and quality of the tracking depends on the amplitude and on frequencies contained in the reference signals. 
However, for such complex reference signals, the simulation and the realization of experimental prototypes are essential tools for testing the possibilities and the performances of our RFCS in order to confirm their usefulness according to the requirements of the application at hand.

\section{References}

[1] Atherton, D. P., "Early developments in nonlinear control", in IEEE Control Systems Magazine, vol. 16, no. 3, pp. 34-43, June 1996, doi: 10.1109/37.506396.

[2] Holmberg, U., "Relay feedback of simple systems", Ph.D. thesis TFRT-1034, Department of Automatic Control, Lund Institute of Technology, Lund, Sweden, 1991.

[3] Tsypkin, Ya.Z., "Relay Control Systems", Cambridge University Press, Cambridge, UK, ISBN: $05212439049780521243902,1984$.

[4] Hamel, B., "A mathematical study of on-off controlled higher order systems", in Proceedings of the Symposium on Nonlinear Circuit Analysis, Polytechnic Institute of Brooklyn, New York, vol. 6, pp. 225-232, 1956.

[5] Boiko, I., "Discontinuous Control Systems, Frequency-Domain Analysis and Design", Birkhäuser Boston, a part of Springer Science+Business Media, LLC, ISBN: 978-0-81764752-0. 2009.

[6] Zaatri, A., Belhour S., "Etude et réalisation d'un hacheur PWM", Revue des Energies Renouvelables, vol. 13, no. 1, pp. 187-198, 2010.

[7] Zaatri, A., Azzizi, N., Rahmani, F., "Minimization of energy losses into a PWM chopper", World Journal of Engineering, vol. 11, no. 4, pp. 441-446, 2014. https://doi.org/10.1260/ 1708-5284.11.4.441, 2014.

[8] Lordejani, S., N., Yazdanpanah., M., J., "Sliding Mode Pulse Width Modulation for Voltage Control of a Voltage Source Inverter", $23^{\text {rd }}$ Iranian Conference on Electrical Engineering, 2015, pp. 1642 - 1646. 